\title{
Gathering Structured Patient Insight to Drive the PRO Strategy in COPD: Patient-Centric Drug Development from Theory to Practice
}

\author{
Francesco Patalano - Florian S. Gutzwiller - Bhavik Shah • \\ Chitresh Kumari · Nigel S. Cook
}

Received: September 3, 2019 / Published online: November 9, 2019

(c) The Author(s) 2019

\begin{abstract}
We illustrate our experience of gathering patient insights on the most patient-relevant symptoms in chronic obstructive pulmonary disease (COPD) via a structured and systematic approach towards 'patient-centric' drug development, leveraging recent advances in digital technologies using online platforms. The fourstep approach comprised the following: literature search, social media listening (SML) study, online bulletin board (OBB) exercise, and design of an online patient preference study (PPS). The initial online studies (SML and OBB) revealed that, besides dyspnoea and exacerbations, patients perceive cough and mucus production as equally important aspects of disease management for COPD. To further build and quantify patients' understanding of the importance of these symptoms, an online patient preference survey is underway. Based on these findings, we have elected to include the Cough and Sputum Assessment Questionnaire or CASA-Q, a
\end{abstract}

Enhanced Digital Features To view enhanced digital features for this article go to https://doi.org/10.6084/ m9.figshare.9995702.

F. Patalano $(\bowtie)$ · F. S. Gutzwiller · N. S. Cook

Novartis Pharma AG, Basel, Switzerland

e-mail: francesco.patalano@novartis.com

B. Shah · C. Kumari

Novartis Healthcare Pvt Ltd, Hyderabad, India validated instrument to collect patient-reported outcomes (PRO), besides the use of the COPD assessment test or CAT to assess the severity and impact of COPD in drug development studies for COPD. Additionally, to capture movement and sleep disturbance, we consider the inclusion of actigraphy as a digital evidence-capture end point. Lastly, in a phase II trial, a survey questionnaire on incontinence will be administered to evaluate the importance of this issue among patients. We believe that integrating insights derived from "online" studies (SML, OBB, and PPS) into drug development offers an opportunity to truly listen to patients' voices in early product design ensuring relevance of end points selected for the clinical trial program. This approach also has the potential to complement conventional qualitative and quantitative data collection requirements for PRO instrument development. While awaiting final guidance from the US Food and Drug Administration, or FDA, the recently released draft documents on collecting representative patients' input reference social media as a tool to collect qualitative patient preference data and these developments suggest that patient preference data can influence future clinical trial design, end point selection, and regulatory reviews.

Funding: Novartis Pharma AG, Basel. 
Keywords: COPD; Drug development; Online bulletin board; Patient perspective; Patient preferences; Social media

\section{Key Summary Points}

Early patient insights are valuable.

Gaining patient insights by collecting information on their perspective is an ongoing process throughout the product's lifecycle.

Integrating patients' insights gathered via a structured and systematic approach, including 'online' studies, is feasible and benefits decision making, if considered early during drug development.

\section{INTRODUCTION}

The healthcare industry is undergoing a paradigm shift towards a more patient-centric approach as patients take centre stage in the contemporary patient-focused drug development (PFDD) process [1, 2]. For successful development of meaningful therapies, it has become vital to determine what matters most to patients living with the respective condition. A growing number of pharmaceutical and device companies are now incorporating patients' perspectives throughout the drug development process. As active patient engagement is increasing, it has become critical to systematize the tools and approaches used to collect patients' perspectives and design a standardized process that can inform drug development based on patient-relevant end points and outcomes. Regulatory authorities do acknowledge the importance of early patient engagement and appreciate and early engagement with authorities on methods to collect patients' insights [3].

The evolution of internet from the static Web to the highly participative and intuitive Web 2.0 has transformed communication channels for patients [4]. Besides seeking traditional sources of healthcare information, patients are increasingly using online health forums to exchange information [5]. Studying such online interactions from social media provides an opportunity to understand expectations of patients related to their disease condition. In addition, in the digital era, it is possible to engage patients virtually without the need for face-to-face interactions.

To leverage the recent advancements in digital technologies and exploit available online platforms to gain insights regarding symptoms that would be most relevant from patients' perspectives in chronic obstructive pulmonary disease (COPD), we considered a four-step approach (Fig. 1, Table 1): (1) literature review, (2) social media listening (SML) study, (3) online bulletin board (OBB), and (4) web-based patient preference study. Steps 1-3 have already been completed, while step 4 is ongoing.

We illustrate our experience with this approach to gathering patient insights in a structured and systematic way from patients with COPD and how we are leveraging such early insights to select relevant patient-reported outcome (PRO) end points to use in prospective clinical program for an early drug development project. This article is based on previously conducted studies and does not report any new data with human participants or animals performed by any of the authors.

\section{PATIENT INSIGHTS THROUGH SOCIAL MEDIA AND ONLINE PLATFORMS}

\section{Literature Review}

The first step in our approach was a targeted literature review, performed to understand published literature regarding patient needs and expectations related to COPD. The findings of this literature review revealed that most of the patient preference studies (15 of the total 22 retrieved studies in COPD) have traditionally evaluated key attributes around device preferences. Only two studies focused on the diseaserelated aspects that patients would most likely 
(a)

Traditional Approach

New approach

(b)

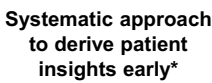

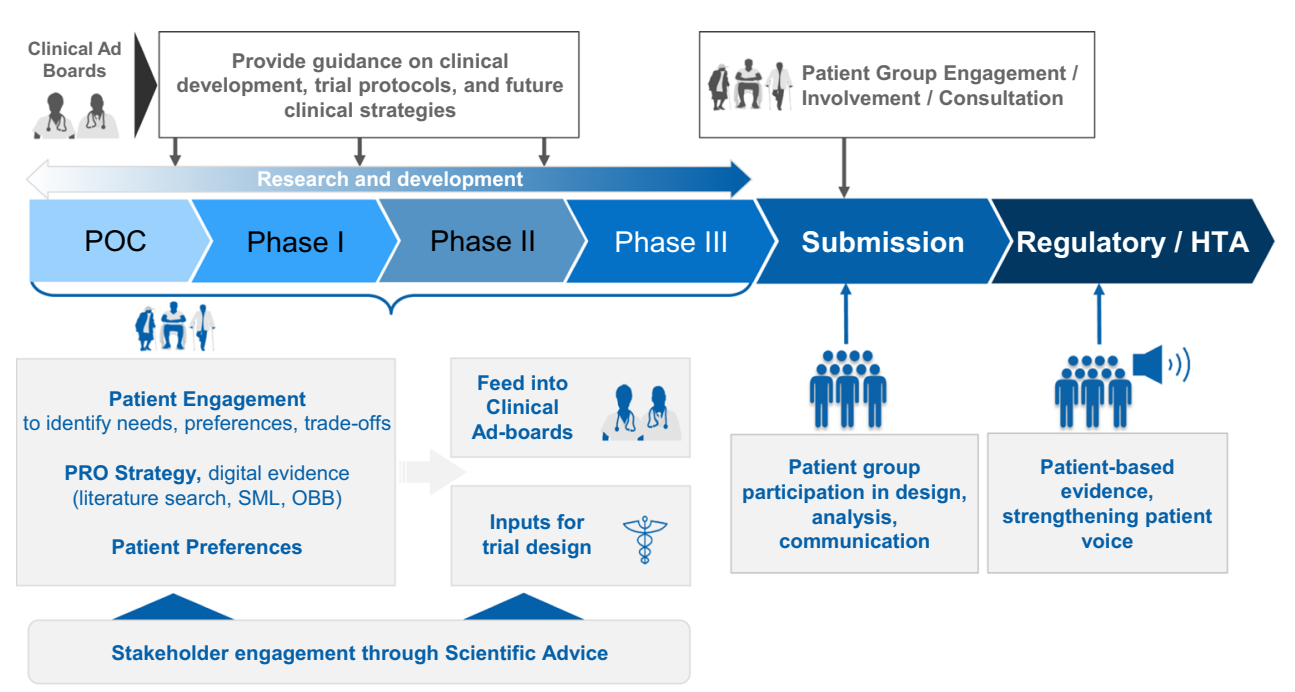

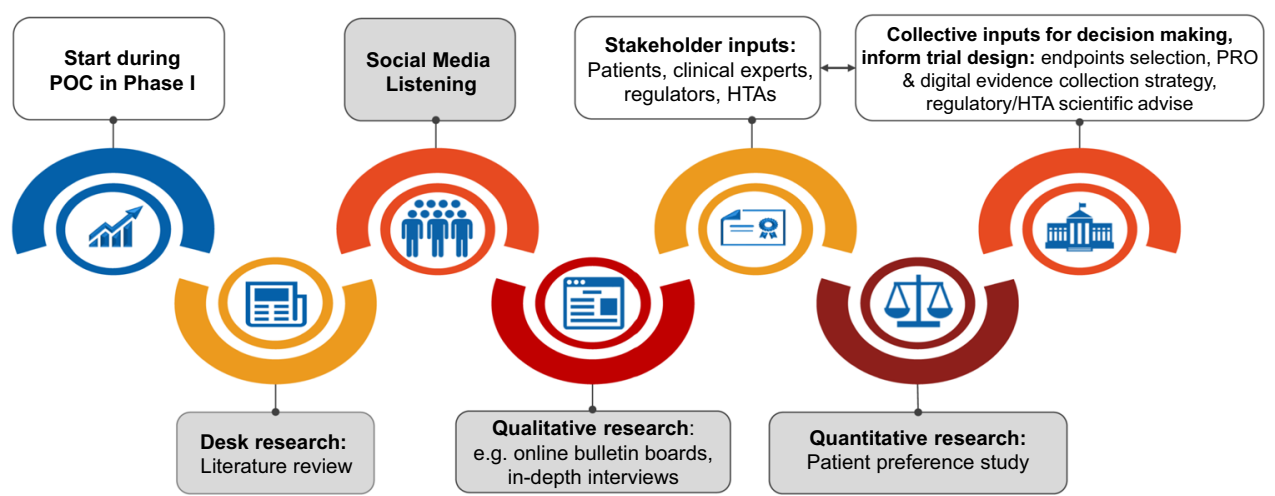

Fig. 1 Paradigm shift: patient involvement throughout development to launch and beyond. The upper part of the figure depicts patient involvement in drug development with evolving patient-centric drug development (a). The lower part illustrates the systematic steps that we used to

to alleviate or on an ideal treatment profile for COPD from the patients' perspective $[9,10]$. This analysis offered us an understanding of the current knowledge gap concerning preferences of COPD patients and provided the basis for developing the search strategy for the subsequent social media analysis.

\section{SML}

The next step was an SML study-an analysis of online conversations in open-access platforms among patients with COPD. Analysis of patient interactions on social media is a quick approach to obtain rich information about patient unmet derive patient insights via early engagement $(\mathbf{b}) .{ }^{*}$ The grey boxes indicate steps with "online" studies considered for the presented case study. $O B B$ online bulletin board, $P O C$ proof of concept, $P R O$ patient-reported outcome, $S M L$ social media listening, HTA health technology assessment

needs, disease management, and delivery of care [11]. Importantly, SML allows researchers to "listen" to the open discussions that are already happening online in the patients' own words, without influencing those conversations and imposing any further research burden on the patient community $[12,13]$. Although it is convenient to gather patient inputs via studying social media postings, there are inherent limitations due to the nature of SML, such as self-selection bias (not everyone engages online; greater likelihood of negative aspects being posted; may include a narrow representation of clinical/demographic characteristics) limiting 
Table 1 Key steps and corresponding questions/objectives [6-8]

\begin{tabular}{ll}
\hline Key steps & $\begin{array}{l}\text { Pertinent questions/objectives to understand relevant patient } \\
\text { perspective }\end{array}$ \\
\hline Step 1: qualitative observational-SML & Observe patient conversations \\
& What affects, what motivates the patients? \\
& What are the questions, pains, experiences, concerns? \\
& How do they communicate about their disease? \\
& Asking specific questions \\
Step 2: qualitative dialogue_-OBB & Explore the disease experience \\
& Understand the priorities \\
& Detect potentially hidden aspects \\
& Further understand the communication \\
& Among all disease aspects \\
Step 3: quantitative research-patient preference & Define priorities: what is important? \\
studies & Which trade-offs can be accepted? \\
& What would make a future product attractive to the patient? \\
\hline
\end{tabular}

$O B B$ online bulletin board, $S M L$ social media listening

generalizability; no verification of identity; and the passive observational nature not allowing further probing to seek clarification on the posted views $[14,15]$. At this time there is a lack of guidance surrounding the key ethical issues such as privacy, anonymity, and informed consent (voluntary participation) that may arise in analysing the content posted on social media [16]. To respect patient privacy during the SML study conduct and reporting, we adhered to the data privacy obligations in accordance with the Health Insurance Portability and Accountability Act (HIPAA) [17]. All the data for SML study were obtained from publicly accessible sources without accessing password-protected information and only aggregated qualitative findings derived based on the anonymized content were reported. The detailed methodology along with results of COPD SML study has been reported elsewhere [6]. As a follow-up to the SML study, it is important to conduct qualitative research studies with patients to confirm and expand on the findings from SML.

\section{$O B B$}

Subsequently we conducted an OBB study to further validate and explore the patient insights revealed from SML in greater detail with a qualitative approach. Twenty COPD patients (UK, $n=10$; USA, $n=10$ ) participated in the OBB that ran for over 2 weeks [7]. Being an asynchronous, moderated, closed online community platform (similar to a private chat room) for qualitative patient research, an OBB allows participants to answer predefined questions in a comprehensive manner [12]. Information derived from the literature search and SML study formed the basis for the questions developed for the OBB. An OBB offers the interactive benefits of a face-to-face focus group discussion, but without certain individuals dominating the conversation. It also has the potential to generate deep insights, including emotional and sensitive aspects of living with a disease, which may not surface in a traditional interview settings $[12,18]$. A key strength of this exercise is that it provides a platform for candid, honest, and shared patient-to-patient interactions while maintaining anonymity through 
the study [12, 19]. When the participating patients were contacted for debriefing at the end of the OBB session, the patients expressed agreement with the conclusions derived from the discussions and also expressed that they were very engaged throughout their participation in the study. The comments shared by patients on the final day of the OBB study reflected that patients really valued the opportunity to participate in this form of research and share experiences with 'like' patients.

The detailed findings from the qualitative online insight gathering approaches (SML and OBB studies) in COPD have been reported in their respective full publications $[6,7]$. In short, the results of these studies indicated that besides dyspnoea and exacerbations, cough and mucus production are very important aspects of the disease for COPD patients. The findings from the SML study showed that cough and mucus production were the symptoms affecting quality of sleep, work, and daily activities irrespective of severity of disease [6]. These findings also revealed that relief from cough, mucus production, and shortness of breath would be the most desirable aspects of disease management from a patient perspective (Table 2) $[6,7]$.

The results from the OBB study conducted with patients suggested that the symptoms of cough and excess mucus production are particular issues for the patients early in the day, with the effort of mucus expulsion leaving them tired and exhausted for the day ahead. These cough and mucus symptoms also result in the need for continuous breaks during the daytime making most patients exhausted. The symptoms also manifest during the night (phlegm accumulates when lying down and introduces sense of suffocation, together with the cough causing sleep disturbance) [7]. Another important observation from the OBB study was that patients with COPD experience urinary incontinence associated with cough but tend to be uncomfortable talking openly about such sensitive symptoms (hence this was not a prominent complaint in social media posts); in fact, two-third of the study participants from OBB who suffered from chronic cough acknowledged incontinence to be a significant problem $[6,7]$. To build further on the significance and relative importance of these symptoms to patients (cough and mucus secretion and consequences thereof such as sleep disturbance, fatigue, incontinence) compared with the more commonly measured end points (lung capacity or breathlessness; exacerbations/hospitalizations) in COPD, there was a need to evaluate them in a quantitative patient preference study.

\section{Web-Based Patient Preference Study}

Patient preferences reflect patients' choices among different disease state alternatives or particular health interventions (drug or medical device) over other available options, based on

Table 2 Key findings from the SML and OBB studies $[6,7]$

\begin{tabular}{ll}
\hline SML & OBB and qualitative interviews \\
\hline $\begin{array}{c}\text { Difference in symptoms discussed online by COPD patients } \\
\text { and other healthcare stakeholders }\end{array}$ & $\begin{array}{c}\text { Relief of cough, mucus production, and shortness of } \\
\text { breath are most desirable aspects from patient } \\
\text { 'Serspectives } \\
\text { mentions by other healthcare stakeholders }\end{array}$ \\
$\begin{array}{c}\text { With patients, 'Cough with or without mucus' was the most } \\
\text { frequently mentioned symptom (27\%), followed by 'mucus }\end{array}$ & $\begin{array}{c}\text { Patients were uncomfortable talking openly about urinary } \\
\text { clearance from lungs' (22\%) and 'shortness of breath' (20\%) }\end{array}$ \\
$\begin{array}{c}\text { incontinence associated with cough } \\
\text { Incontinence was mentioned very infrequently in online posts }\end{array}$ & $\begin{array}{c}\text { All these symptoms have a big social and emotional } \\
\text { impact }\end{array}$ \\
\hline
\end{tabular}

$C O P D$ chronic obstructive pulmonary disease, $O B B$ online bulletin board, $S M L$ social media listening 
trade-offs between the advantages and disadvantages of the preferred choice $[20,21]$. To make a choice or stated preference, patients need to weigh the advantages and disadvantages of each disease state or intervention and compare them with the alternatives. The methods to derive patient preferences focus on understanding the relative importance of attributes by patients and provide an alternative to characterize patient needs and desires [21]. We are evaluating the findings from the SML and OBB studies in a quantitative patient preference study. This study is currently ongoing via a discrete-choice experiment (DCE) online survey in patients with COPD, which will further quantify the relative importance of different symptoms from the patients' perspective.

While designing the preference study we approached a health technology assessment (HTA) agency to obtain input on study designa first of its kind 'scientific advice' from an HTA body. We sought input from NICE (The National Institute for Health and Care Excellence) on the COPD patient preference study design and incorporated many suggestions received through this early scientific advice into the study protocol [22]. In particular, considering the recommendations by NICE, the study protocol was updated for eligibility criteria, quality of life questionnaire instrument selection, and total number of patients to be recruited for selected countries.

The patient preference study will use a DCE design, built on the principle of random utility theory, that assumes that disease states can be decomposed into various attributes (symptoms) and the attractiveness/unattractiveness of a disease state depends on patients' relative preferences for the attributes (symptoms) expressed by the frequency with which they choose their preferred disease profile [23]. In our DCE survey, patients with COPD will be asked to choose between two disease states with varying levels of the aforementioned attributes, as expressed by their willingness to accept trade-offs among them [24]. This 'disease state' preference survey is a fairly novel approach as typically the discrete choice is made between two (hypothetical) treatment options. We believe the choice among disease states is a more appropriate exercise for patients when investigating the relative importance of symptoms to them, as it avoids any confusion between attributes/symptoms being incorrectly interpreted as side effects of a product. The results of this ongoing preference study will provide estimates of the relative importance of different symptoms to patients, which in turn will provide evidence about the importance of incorporating these patient-relevant end points in clinical trials. Furthermore, the results will provide a basis for determining the relative value, from the patients' perspective, that future treatments would offer that address these same symptoms to a greater or lesser degree.

\section{INTEGRATION OF COLLECTIVE INSIGHTS TO INFORM CHOICE OF PRO END POINTS}

The integration of insights derived from "online" studies (SML, OBB, and the online patient preference study) into drug development for COPD offers an opportunity to truly listen to patients' voices and inform drug development strategy and product design as a step towards a more PFDD-based model. The insights generated from these studies will be of greatest value for taking into account patients' needs and preferences while defining clinical studies in COPD. Collectively, these patient insights and preferences will help assemble hypothetical treatment profiles with specific characteristics and also aid in selecting clinical outcome assessments beyond conventional end points in the COPD drug development program.

Having identified what is important for patients would not serve the purpose until these elements are appropriately integrated into a clinical trial setting in a timely fashion (e.g., identifying relevant end points, selecting an appropriate PRO instrument, and device 'digital' evidence capture strategy). Timely integration of the findings from the patient preference study into drug development is vital for improving (patient-relevant) outcomes in COPD patients. Traditionally, the phase III clinical trials evaluating efficacy and safety of drugs for COPD have been using clinical 
measures such as FEV1 and exacerbations. We considered the insights derived from qualitative and quantitative studies such as SML, OBB, to inform PRO end point selection. Based on the findings derived from the patient insight studies, it appears that cough and mucus production are additional symptoms where patients with COPD would value new therapeutic options. Therapeutic management of patients with COPD has evolved towards more tailored and individualized treatment from the past where a common therapeutic approach was used for all patients [25]. The role of a more individualized approach for management of COPD has been an emerging strategy for personalized therapy in COPD [26]. A careful evaluation of individual patient needs can lead to improved use of available treatment options and support precision medicine when there is concordance between genotype and phenotypic manifestations of the diseases [26, 27]. With advancement of the therapies that may address symptoms such as cough and mucus in the future, it will be an important step forward for the patient subgroup suffering from these symptoms to have new treatment options to complement the use of bronchodilators and corticosteroids. Using rigorously collected patient insights and the newly available treatment options, coupled with enhanced diagnostic techniques, may result in better outcomes for the individual patients and the overall population. Consequently, it will be appropriate to use a PRO assessment that evaluates the impact of cough and mucus production in the prospective clinical trial for COPD.

We therefore, intend to use the available Cough and Sputum Assessment Questionnaire (CASA-Q) [28], a validated instrument to collect PROs, in the prospective drug development studies for COPD in addition to the use of the COPD assessment test (CAT) to assess the severity and impact of COPD. The preference study results will further illustrate the importance of the other consequences of cough and mucus-sleep disturbance, fatigue, and incontinence, for instance-to patients to justify their inclusion as standard PRO measures in prospective COPD clinical trials. We are considering inclusion of actigraphy as a digital end point in clinical trials to capture movement and sleep disturbances. During the phase II trial, a survey questionnaire on incontinence will be administered to patients to further evaluate the importance of this issue among patients. Once we have the final outputs of the preference study, we also intend to solicit regulatory and HTA scientific advice to inform the design of future clinical studies in COPD and the insights and preferences of patients will form an important input to that advice meeting.

Regulatory guidance recommends the use of a validated PRO instrument in support of a labelling claim, if such an instrument exists, to assess and measure the concepts of interest [29]. In circumstances where patient insights reveal requirements that cannot be assessed using existing tools or otherwise require the development of a new instrument, then it is necessary to follow the formal approach of developing a new PRO instrument recommended by the regulatory agencies. Developing a PRO instrument is an iterative process $[29,30]$. PRO instrument development requires qualitative and quantitative studies to identify items and domains of an instrument that are appropriate and comprehensive relative to the intended measurement concept, population, and use [30]. We believe that the approach illustrated here has the potential to complement both qualitative (via SML and OBB studies) and quantitative (by patient preference studies) data collection requirements during the PRO instrument development process. Importantly, if aimed to use for PRO instrument development, such studies need to begin very early in the drug development lifecycle (at latest in phase I) so that the instruments can be validated in phase II to be ready by the start of the pivotal clinical trials for registration.

\section{FUTURE DIRECTIONS}

Regulatory agencies such as the Center for Devices and Radiological Health (CDRH) at the US Food and Drug Administration (FDA) advocate early engagement and frequent discussions regarding design and execution of patient preference studies [31]. Guidance for the 
incorporation of patient preference measurements into drug development, regulatory, and reimbursement decision-making processes is evolving [3, 32] and multi-stakeholder initiatives such as IMI PREFER (Innovative Medicines Initiative's The Patient Preferences in BenefitRisk Assessments during the Drug Life Cycle project) are actively working to fill this gap [32]. There is a need for early engagement in dialogue with regulatory agencies and HTA bodies on scientific advice to discuss patient needs, preferences, and implications thereof for clinical trial design and evidence collection [8]. This step is crucial since, eventually, HTA agencies will be one of the recipients of any product submissions that will be developed through trials using the insights generated through such a PFDD exercise and would need to make reimbursement decisions after recognizing the value that such products offer to patients.

The draft FDA guidance advocates incorporating the patients' voice via collection of patient experience data and other relevant information to leverage the expertise that patients and caregivers can bring to the PFDD model [33]. This FDA draft guidance details data collection methodologies for patient experience data (including social media to collect qualitative data), with an intention to inform clinical trial design and trial end point selection and to facilitate regulatory reviews. The guidance, however, mentions that "the level of rigor needed to generate such data" will differ across studies depending on the clinical condition and treatment under investigation and encourages early FDA consultation to obtain feedback [33]. Although the current draft is undergoing stakeholder review and consultation, the availability of this guidance indicates that regulatory agencies encourage stakeholders to explore innovative tools to gain insights into patients' perspectives related to disease burden. The FDA held a workshop intended to develop more guidance documents on methods to identify what is important to patients and select, develop, or modify fit-for-purpose clinical outcome assessments [34, 35]. The final guidance when available from the FDA will clarify how to best integrate patient insights into the PFDD model.

\section{CONCLUSIONS}

Gaining patient insights by collecting information on their disease experiences and perspectives should be an ongoing process throughout the product development lifecycle and starts in very early development phases. Conducting "online" studies such as the SML, OBB, and online patient preference studies is a relatively new way to generate patient insights. Integrating patient insight gathered via structured and systematic approaches including such "online" studies is feasible and an important input to decision making if considered early during drug development.

\section{ACKNOWLEDGEMENTS}

Funding. Article processing charges (Rapid Service and Open Access Fees) were funded by Novartis Pharma AG, Basel.

Authorship. All named authors meet the International Committee of Medical Journal Editors (ICMJE) criteria for authorship for this article, take responsibility for the integrity of the work as a whole, and have given their approval for this version to be published.

Authors' contributions. FP and NSC conceptualized the idea with inputs from FSG, BS, and CK. BS prepared the first draft of manuscript with inputs from all authors; all authors contributed to the interpretation and revision of subsequent drafts and reviewed and approved the final version before submission.

Disclosures. Francesco Patalano is a fulltime employee of Novartis and has Novartis stock(s)/stock options. Florian S. Gutzwiller is a full-time employee of Novartis and has Novartis stock(s)/stock options. Bhavik Shah is a fulltime employee of Novartis. Chitresh Kumari is a full-time employee of Novartis. Nigel S. Cook is a full-time employee of Novartis and has Novartis stock(s)/stock options. The views and opinions expressed in this article are those of 
the authors and do not necessarily represent the views of Novartis.

Compliance with Ethics Guidelines. This article is based on previously conducted studies and does not report any new data with human participants or animals performed by any of the authors.

Data Availability. Data sharing is not applicable to this article as no datasets were generated or analyzed during the current study.

Open Access. This article is distributed under the terms of the Creative Commons Attribution-NonCommercial 4.0 International License (http://creativecommons.org/licenses/ by-nc/4.0/), which permits any noncommercial use, distribution, and reproduction in any medium, provided you give appropriate credit to the original author(s) and the source, provide a link to the Creative Commons license, and indicate if changes were made.

\section{REFERENCES}

1. Borup G, Bach KF, Schmiegelow M, Wallach-Kildemoes $\mathrm{H}$, Bjerrum OJ, Westergaard N. A paradigm shift towards patient involvement in medicines development and regulatory science: workshop proceedings and commentary. Ther Innov Regul Sci. 2016;50(3):304-11.

2. Lowe MM, Blaser DA, Cone L, Arcona S, Ko J, Sasane $\mathrm{R}$, et al. Increasing patient involvement in drug development. Value Health. 2016;19(6):869-78.

3. Haerry D, Landgraf C, Warner K, Hunter A, Klingmann I, May $M$, et al. EUPATI and patients in medicines research and development: guidance for patient involvement in regulatory processes. Front Med. 2018. https://doi.org/10.3389/fmed.2018. 00230 .

4. Hesse BW, O'Connell M, Augustson EM, Chou W-YS, Shaikh AR, Rutten LJF. Realizing the promise of web 20 : engaging community intelligence. J Health Commun. 2011;6(Suppl 1):10-31. https:// doi.org/10.1080/10810730.2011.589882.

5. Smailhodzic E, Hooijsma W, Boonstra A, Langley DJ. Social media use in healthcare: a systematic review of effects on patients and on their relationship with healthcare professionals. BMC Health Serv Res. 2016;16(1):442. https://doi.org/10. 1186/s12913-016-1691-0.

6. Cook NS, Kostikas K, Gruenberger JB, Shah B, Pathak P, Kaur VP, et al. Patients' perspectives on COPD: findings from a social media listening study. ERJ Open Res. 2019;5(1):00128-2018. https://doi. org/10.1183/23120541.00128-2018.

7. Cook N, Gey J, Oezel B, Mackay AJ, Kumari C, Kaur $\mathrm{VP}$, et al. Impact of cough and mucus on COPD patients: primary insights from an exploratory study with an online patient community. Int J Chronic Obstr Pulm Dis. 2019;14:1365.

8. Cook NS, Cave J, Holtorf A-P. Patient preference studies during early drug development: aligning stakeholders to ensure development plans meet patient needs. Front Med. 2019. https://doi.org/10. 3389/fmed.2019.00082.

9. Svedsater H, Roberts J, Patel C, Macey J, Hilton E, Bradshaw L. Life impact and treatment preferences of individuals with asthma and chronic obstructive pulmonary disease: results from qualitative interviews and focus groups. Adv Ther. 2017;34:1466-81.

10. Pisa G, Freytag S, Schandry R. Chronic obstructive pulmonary disease (COPD) patients' disease-related preferences. Patient. 2013;6(2):93-101.

11. Street J, Farrell L. Analysis of Social Media. In: Facey $\mathrm{K}$, Hansen $\mathrm{H}$, Single A, editors. Patient involvement in health technology assessment. Singapore: Springer Nature; 2017. p. 175-85.

12. Holtorf AP, Cook N. The role of patients in market access. In: Kockaya G, Wertheimer A, editors. Pharmaceutical market access in developed markets. SEED Open Books; 2018. pp. 267-88. https:// doi.org/10.7175/747.ch18.

13. Mikolajczak C. Social media listening: uncovering patients' needs. 2017. https://pharmaphorum.com/ views-and-analysis/social-media-listeninguncovering-patients-needs/. Accessed 21 Aug 2019.

14. Grajales III FJ , Sheps S, Ho K, Novak-Lauscher H, Eysenbach G. Social media: a review and tutorial of applications in medicine and health care. J Med Internet Res. 2014;16(2):e13.

15. Moorhead SA, Hazlett DE, Harrison L, Carroll JK, Irwin A, Hoving C. A new dimension of health care: systematic review of the uses, benefits, and limitations of social media for health communication. J Med Internet Res. 2013;15(4):e85. https://doi.org/ 10.2196/jmir.1933. 
16. Hunter RF, Gough A, O'Kane N, McKeown G, Fitzpatrick A, Walker $\mathrm{T}$, et al. Ethical issues in social media research for public health. Am J Public Health. 2018;108(3):343-8. https://doi.org/10. 2105/ajph.2017.304249.

17. El Emam K, Rodgers S, Malin B. Anonymising and sharing individual patient data. Br Med J. 2015. https://doi.org/10.1136/bmj.h1139.

18. Guest G, Namey E, Taylor J, Eley N, McKenna K. Comparing focus groups and individual interviews: findings from a randomized study. Int J Soc Res Meth. 2017;20(6):693-708.

19. Cook NS, Tripathi P, Weiss O, Walda S, George AT, Bushell A. Patient needs, perceptions, and attitudinal drivers associated with obesity: a qualitative online bulletin board study. Adv Ther. 2019; 36(4):842-57. https://doi.org/10.1007/s12325-01900900-1.

20. US Food and Drug Administration: Patient preference information-voluntary submission, review in premarket approval applications, humanitarian device exemption applications, and de novo requests, and inclusion in decision summaries and device labeling. 2016. https://www.fda.gov/ downloads/medicaldevices/deviceregulationandgui dance/guidancedocuments/ucm446680.pdf. Accessed 28 Aug 2018.

21. Bridges J, Onukwugha E, Johnson F, Hauber A. Patient preference methods-a patient centered evaluation paradigm. ISPOR Connect. 2007;13(6): 4-7.

22. NICE. NICE provides first scientific advice on patient preference study design. https://www.nice. org.uk/news/article/nice-provides-first-scientific-ad vice-on-patient-preference-study-design. Accessed 26 Aug 2019.

23. Lancaster KJ. A new approach to consumer theory. J Polit Econ. 1966;74(2):132-57.

24. Liu FX, Witt EA, Ebbinghaus S, DiBonaventura Beyer G, Shinde R, Basurto E, et al. Patient and oncologist preferences for attributes of treatments in advanced melanoma: a discrete choice experiment. Patient Prefer Adherence. 2017;11:1389-99. https://doi.org/10.2147/PPA.S140226.

25. Franssen FM, Alter P, Bar N, Benedikter BJ, Iurato $\mathrm{S}$, Maier D, et al. Personalized medicine for patients with COPD: where are we? Int J Chron Obstr Pulmon Dis. 2019;14:1465.

26. Sidhaye VK, Nishida K, Martinez FJ. Precision medicine in COPD: where are we and where do we need to go? Eur Respir Rev. 2018;27(149):180022.
27. Agusti A, Calverley PM, Celli B, Coxson HO, Edwards LD, Lomas DA, et al. Characterisation of COPD heterogeneity in the ECLIPSE cohort. Respir Res. 2010;11(1):122.

28. Crawford B, Monz B, Hohlfeld J, Roche N, Rubin B, Magnussen $H$, et al. Development and validation of a cough and sputum assessment questionnaire. Respir Med. 2008;102(11):1545-55. https://doi.org/ 10.1016/j.rmed.2008.06.009.

29. Guidance for industry patient-reported outcome measures: use in medical product development to support labeling claims US Food Drug Administration. 2009. https://www.fda.gov/downloads/drugs/ guidances/ucm193282.pdf. Accessed 28 Aug 2018.

30. Turner RR, Quittner AL, Parasuraman BM, Kallich JD, Cleeland CS. Patient-reported outcomes: instrument development and selection issues. Value Health. 2007;10(Suppl 2):S86-93. https://doi. org/10.1111/j.1524-4733.2007.00271.x.

31. US Food and Drug Administration: Patient preference information-voluntary submission, review in premarket approval applications, humanitarian device exemption applications, and de novo requests, and inclusion in decision summaries and device labeling. Guidance for Industry, Food and Drug Administration Staff and Other Stakeholders In: CDRH, editor. Silver Spring: Office of Communication, outreach and Development; 2016.

32. de Bekker-Grob EW, Berlin C, Levitan B, Raza K, Christoforidi K, Cleemput I, et al. Giving patients' preferences a voice in medical treatment life cycle: the PREFER public-private project. Patient. 2017;10(3):263-6. https://doi.org/10.1007/s40271017-0222-3.

33. US Food and Drug Administration: Patient-focused drug development: collecting comprehensive and representative input-guidance for Industry, Food and Drug Administration staff, and other stakeholders [draft guidance]. https://www.fda.gov/ downloads/drugs/guidancecomplianceregulatoryin formation/guidances/ucm610442.pdf. Accessed 26 Oct 2018.

34. US Food and Drug Administration: Patient-focused drug development public workshop on guidance 2: methods to identify what is important to patients. http://www.fda.gov/downloads/Drugs/NewsEvents/ UCM620707.pdf. Accessed 26 Oct 2018.

35. US Food and Drug Administration: Patient-focused drug development public workshop on guidance 3: select, develop or modify fit-for-purpose clinical outcomes assessments. https://www.fda.gov/down loads/Drugs/NewsEvents/UCM620708.pdf. Accessed 26 Oct 2018. 